\section{BAMBINI CHE (NON) VANNO A SCUOLA: PRIMA E DOPO IL COVID}

Parlare di Scuola oggi ci obbligherebbe in qualche modo a parlarne bene. La chiusura della Scuola, strumento di prevenzione alla diffusione della pandemia, pone la collettività ad animarsi nel conflitto salute-educazione polarizzandosi forse in modo eccessivo. In questo clima, eserciti di genitori e un po' meno di alunni reclamano la riapertura, tecnici e politici dibattono su quanto la chiusura sia la giusta scelta di Sanità pubblica, mentre gli addetti alla Salute mentale, di grandi e piccoli, iniziano a registrare l'impatto di un drastico cambiamento nelle abitudini di bambini, adolescenti e intere famiglie. Precedentemente alla pandemia il conflitto salute-educazione poteva essere diversamente interpretato: movimenti di alunni che rivendicando il diritto alla salute del pianeta rinunciavano alla frequentazione della Scuola. Questa pluralità di visioni sul tema Scuola suggerisce un approccio cauto e aperto. È quindi di estrema utilità la lettura dell'articolo proposto in questo numero (pag. 232) che descrive e analizza i dati legati al fenomeno dell'assenteismo scolastico e dell'abbandono scolastico'.

Partiamo quindi da questi fenomeni, oggi apparentemente anacronistici, ma in realtà attuali nelle proiezioni delle traiettorie didattiche e centrali nella dialettica salute-educazione.

\section{La Scuola nella traiettoria evolutiva del bambino}

Tracciando una linea, con una penna, senza mai sollevarla dal foglio, riproduciamo in modo figurato un percorso di crescita composto dall'insieme di minimi passaggi evoluti$\mathrm{vi}$, individuabili come minuscoli punti, ma che uniti in una soluzione di continuità forniscono il senso dell'intero, di quello che è stato e di quello che è. Nella vita dell'individuo, $\mathrm{i}$ passaggi iniziali, quelli che appartengono all'infanzia, si concentrano ammassati in momenti di piena evoluzione (tappe motorie, del linguaggio, comunicative, sociali), creando quindi una linea spessa che andrà con il tempo diradandosi in un tratto fine segnato da punti assai significativi (autonomia, indipendenza, responsabilità crescenti). Immaginiamo ora i compiti evolutivi da affrontare nel contesto scolastico in continuità con quelli che li precedono nel periodo della prescolarità.

Riflettendo questa visione sul fenomeno dell'assenteismo e della dispersione scolastica potremmo porre una riflessione: quali sono oggi i traguardi evolutivi mancati e mancanti, separazioni nella linea tracciata, che nella storia di alcuni bambini e adolescenti non sono stati compiuti e quindi non hanno preparato al passaggio successivo?

In questo ragionamento esulano probabilmente episodi a carattere traumatico e che sono direttamente correlabili con il ritiro scolastico o con l'assenteismo (malattia, bullismo, violenza, abuso, lutti ecc.) la cui potenza emotiva è tale da pervadere i processi cognitivi già acquisiti.

Gli elementi che preparano alla Scuola si consumano nel contesto familiare e domestico, nel contesto sociale e culturale. I prerequisiti scolastici non sono infatti riferibili ai soli processi cognitivi della letto-scrittura o del fare di calcolo. In una versione accademica dei generici prerequisiti scolastici possiamo citare l'adeguato rapporto con il pari, il rapporto con l'adulto di riferimento, l'essere parte di un gruppo, il rispetto della regola, il rispetto del turno, la comprensione del contesto, la gestione dello spazio ecc. Con approccio evolutivo potremmo asseverare che ancor prima è necessario che ci siano presupposti che abituino il bambino a emozionarsi nelle relazioni e nell'apprendimento, a divertirsi nel conoscere e a incuriosirsi nel crescere. Nella prospettiva di superamento e intervento sull'assenteismo scolastico sarà possibile intervenire già in epoca prescolare. Il pediatra potrà e dovrà schierarsi con i bambini, per comprenderne i bisogni evolutivi facilitandone la condivisione con i genitori.

\section{Il ruolo della Scuola: istruire o educare?}

Quanto appena detto potrebbe portare a correlare il fenomeno dell'assenteismo scolastico a mancanze nelle relazioni di crescita. Tutt'altro, ciò che di buono si costruisce nelle relazioni talvolta vale come un seme nella roccia quando la richiesta del contesto risulta involvente. Il contesto Scuola, che involvente non lo è ancora, è ormai colpito da una profonda crisi identitaria. E sul senso della Scuola che poniamo questioni che potremmo pretensiosamente definire istituenti.

Nel secondo dopoguerra, il processo di scolarizzazione dell'Italia, anche in contesti di periferia e di estrema povertà, associata a uno slanciato sviluppo industriale e quindi socio-economico, coinvolgeva l'istituzione scolastica nel processo di emancipazione e crescita del Paese. Un successivo periodo storico caratterizzato dai movimenti di lotta sociale negli anni '70 animava critiche nei confronti della stessa Scuola, contrapponendo il senso pedagogico, educativo e formativo della Scuola a una didattica autoritaria, dove gli insegnamenti, contenuti predisposti andavano a riempire i contenitori passivi, gli alunni. II significato della Scuola e il dibattito a esso correlato con il tempo si è evoluto, rimanendo però ancora oggi aperta l'annosa questione: se luogo educativo e di crescita, quindi solerte ai processi evolutivi dell'individuo, o se invece luogo dell'istruzione, su contenuti e tecnica.

Non si tratta di una mera dissertazione teorica: gli alunni sono esposti non al confronto ma al giudizio tra pari, la competizione talvolta è abusata, le prestazioni che vengono misurate, confrontate e giudicate possono risultare l'unico indicatore di funzionamento, orientando l'Istituzione verso una deriva tecnocratica. In questo clima, quale spazio è occupato alla cura dei piccoli ma frequenti processi evolutivi che compongono la linea di crescita del bambino e delI'adolescente? Qual è il tempo che si vuole e si può dedicare a chi rimane indietro perché con fragilità adattive? Che continuità potremmo dare alle faticose conquiste in materia di inclusione scolastica? Qual è il tempo concesso a chi tra un passaggio e l'altro ha perso anche una sola tappa? In questo clima come si può recuperare il passo senza innescare sentimenti di inadeguatezza o vergogna da parte delle famiglie e degli alunni?

\section{Scuola e Covid: un'occasione da non perdere}

Ciò che oggi accade in periodo di pandemia, con la sospensione delle lezioni prima e l'introduzione della didattica a distanza poi, offre la possibilità di allargare ulteriormente lo spazio di riflessione sul ruolo della Scuola ${ }^{2}$. 
Ciò che è avvenuto nel corso della chiusura scolastica per gli adolescenti con difficoltà adattiva semplifica ulteriormente i concetti già espressi. In un recente studio ${ }^{3}$ sono stati confrontati indicatori di malessere psicologico (dolori somatici, ansia sociale, depressione, tensione e irrequietezza) tra due gruppi di adolescenti, con e senza storia di disturbo da sintomi somatici: 58 ragazzi con pregressa diagnosi di disturbo da sintomi somatici e 57 coetanei senza storia neuropsichiatrica. L'analisi condotta con due test psicometrici somministrati da remoto, Multidimensional Anxiety Scale for Children Self Report (MASC-2R) e Children's Depression Inventory Short Form (CDI 2-SF), ha messo in luce che i giovani con diagnosi di disturbo somatoforme registravano, negli indicatori di malessere sopraelencati, valori significativamente inferiori rispetto ai coetanei del gruppo di controllo. Sebbene non sia possibile attribuire diretti rapporti di causalità tra chiusura scolastica e miglioramento clinico, possiamo invece riconoscere nei tentativi di adattamento che si consumano in larga misura nelI'ambiente scolastico i possibili generatori di sofferenza. Questi dati confermano la necessità di porre maggiore cura ai processi evolutivi nell'ambiente Scuola, che quando diventa luogo di malessere, e in particolare quando ciò avviene in soggetti con anche temporanee fragilità evolutive, facilita fenomeni discriminatori e di dis-integrazione scolastica. Ed è poi su questi elementi che, successivamente, pediatri, psicologi e neuropsichiatri sono coinvolti in un faticoso intervento di contrasto all'assenteismo cronico e alI'abbandono scolastico, come ben descritto nell'articolo di Cozzi e coll. in questo numero di Medico e Bambino'.

Proseguendo l'esperienza didattica nel contesto della pandemia abbiamo familiarizzato con il nuovo strumento $\mathrm{d}^{\prime} \mathrm{in}$ segnamento, la didattica a distanza (DAD).

Lo schermo, surrogato alla presenza sensoriale sui banchi di Scuola, risulta limitante nel coinvolgere esperienzialmente gli alunni, nell'emozionarli, nel farli crescere nelle relazioni. Manca così materialmente e drammaticamente lo spazio fisico per poter tracciare un segmento del percorso di crescita, lo stesso sul quale si sviluppano i compiti evolutivi.

In conclusione, questa finestra temporale che stiamo vivendo, futuristica e distopica, potrebbe (dovrebbe) rianimare il dibattito sui mezzi e sui fini dell'istituzione scolastica. Come professionisti della salute non dovremmo ora perdere l'occasione di ripensare all'Istituzione scolastica contrastando i processi che impattano sulla salute mentale dei nostri ragazzi.

E allora riconosciamo adesso, subito, i sacrifici compiuti dai nostri giovani a favore della salute degli adulti e orientiamo i decisori nel ridefinire una Scuola che rispetti i bisogni evolutivi dell'individuo e della comunità, liberando I'istituzione da un pericoloso sbilanciamento verso una sola funzione di istruzione e addestramento: "i bambini stanno perversamente soffrendo a beneficio degli adulti" ${ }^{\prime 4}$.

\section{Bibliografia}

1. Cozzi G, Barbi E, Ventura A. Il bambino che non va a scuola: I'assenteismo diagnostico come indizio per il pediatra. Medico e Bambino $2021 ; 40(4)$ :232-6. doi: 10.53126/MEB40232.
2.Tamburlini G, Marchetti F. Pandemia di Covid-19: motivazioni e indicazioni per l'apertura degli spazi educativi per i bambini. Medico e Bambino 2020;39:301-4.

3. De Nardi L, Abbracciavento G, Cozzi G, et al. Adolescents with somatic symptom disorder experienced less anxiety and depression than peers during Covid-19 lockdown. Acta Paediatr 2021 , in process.

4. Green P. Risks to children and young people during Covid-19 pandemic. BM 2020 Apr 28;369:m 1669. doi: 10.1136/bmi. m 1669 .

Giuseppe Abbracciavento

Unità Operativa Complessa di Neuropsichiatria Infantile IRCCS Materno-Infantile "Burlo Garofolo", Trieste

\section{SEQUELE POST-ACUTE DA SARS-COV-2 (PASC): OVVERO IL LUNGO INVERNO DEL COVID-19}

Ancora una volta, partiamo dalla Storia. Da quella della pandemia che più spesso abbiamo confrontato a questa attuale, partiamo cioè dall'influenza Spagnola. Per ragioni di guerra, o per una fisiologica reazione di rinascita, avevamo tentato di dimenticare la pandemia Spagnola. Tanto che non sappiamo ancora con certezza se l'ondata di disturbi neurologici con parkinsonismo (la cosiddetta encefalite letargica del film Risvegli, ispirato all'omonimo libro di Oliver Sacks') sviluppatasi in sovrapposizione epidemiologica con la pandemia influenzale fosse o meno a questa collegata. Ammesso che lo fosse, si sarebbe trattato comunque di una sequela rara, è bene dirlo, ma quando un'infezione ha diffusione epidemica anche il raro diventa a suo modo frequente, a suo modo motivo di preoccupazione. Come preoccupava la panencefalite subacuta sclerosante che poteva seguire l'infezione da morbillo, anche questa rara in termini percentuali, ma rimarcabile in funzione del denominatore particolarmente ampio di soggetti che venivano infettati ogni anno.

In base a queste considerazioni, non sembra poi strano che anche il Covid-19 possa essere seguito da rare e spiacevoli soprese, forse legate al coinvolgimento encefalitico che talora è stato dimostrato in fase acuta di malattia, e in parte sovrapponibili a quelle segnalate anche per i due famosi coronavirus precedessori ${ }^{2}$. Tuttavia, non è sempre facile distinguere gli effetti biologici dell'infezione dalle conseguenze esistenziali di una lunga ospedalizzazione (un terzo dei dimessi a un mese presenta sintomi da disturbo posttraumatico da stress o disturbo d'ansia e quasi la metà soffre di insonnia) ${ }^{3}$, della stanchezza epidemica, delle privazioni dei lockdown, dalla riscoperta della nostra mortalità e fragilità umana, dagli squilibri generazionali acuiti ma non esplicitamente affrontati, dall'incertezza del futuro. In questo contesto, il presidente della Repubblica Mattarella ha recentemente fatto propria la storica esortazione di De Gasperi secondo cui "non abbiamo il diritto di disperare", cioè di togliere ai giovani la speranza del futuro, soprattutto in tempi di crisi come quello attuale. Ed è forse superfluo sottolineare che per giovani oggi intendiamo una popolazione piuttosto ampia che dall'adolescenza abbraccia due o tre decadi di età. L'astenia postinfettiva, la depressione, i cosiddetti disturbi da amplificazione del dolore come la fibro- 
mialgia, sono tutte esperienze fluide per ogni medico: non $c^{\prime}$ è dubbio che la malattia protratta possa costituire sia dal punto di vista organico sia da quello mentale un momento di rottura di equilibri; non è raro riscontrare, soprattutto in adolescenti, patologie funzionali esordite dopo un'infezione prolungata. Poco importa se il fattore biologico dell'infezione abbia o meno giocato un ruolo dimostrabile nell'esordio del disturbo. Quello che, almeno in Pediatria, sembra abbastanza chiaro è che la risposta a questo tipo di sindromi deve essere in prima istanza comportamentale. La ripresa dell'attività motoria, la proposta di nuove attività motivanti, la ripresa in mano del "timone" di se stessi, anche facendo leva sulla particolare plasticità del cervello adolescente $^{4}$, permettono di solito di ripartire e superare il problema. Non sempre, purtroppo, questo è sufficiente. A volte questi disturbi mettono solo in luce turbamenti mentali che erano già presenti, anche se poco espressi, e in questi casi un aiuto neuropsichiatrico può diventare fondamentale. Non sappiamo quanto questo valga anche per la fatica, le sensazioni di "nebbia cerebrale", la febbricola, i dolori muscoloscheletrici che sono a volte riferiti in seguito all'infezione da SARS-CoV-2, tanto da giustificare il riferimento ad appositi ambulatori post-Covid (ma forse è il curante che dovrebbe in prima istanza farsene carico). Non sappiamo quanto di questo stato corrisponda a quello che una volta descrivevamo semplicemente come "convalescenza" e quanto invece possa effettivamente essere spia di nuove malattie.

Sta di fatto che l'infezione da SARS-CoV-2 può essere spesso seguita da sequele biologicamente oggettivabili, con una latenza degli indici di flogosi, o una positivizzazione di autoanticorpi, o ancora una ridotta funzionalità polmonare ${ }^{5,6}$. Anche le manifestazioni simili a geloni che abbiamo osservato a distanza di qualche settimana dai picchi epidemici, e che in alcuni ragazzi persistono per mesi, sono manifestazioni chiaramente oggettivabili, certamente su base vasculopatica ${ }^{7}$. Tra questi casi è emblematico quello di una ragazza che su uno dei due piedi affetti da questi geloni sviluppa improvvisamente una tumefazione con cute fredda, ipersensibilità dolorosa e impotenza funzionale, tanto da impedire la deambulazione senza l'ausilio delle stampelle. Il quadro è quello di una algoneurodistrofia (più modernamente complex regional pain syndrome), che è una delle sindromi da amplificazione del dolore cui si accennava sopra. Già, perché amplificazione non significa finzione, co- me talora si sarebbe portati a pensare. Amplificazione significa entrare in un circolo vizioso squisitamente ed evidentemente psico-organico. La suggestione, il training alI'autocontrollo del sintomo, insieme a una dose di bifosfonato, sono stati sufficienti a "risvegliare" e sgonfiare il piede e quindi gettare via le stampelle, permettendo la ripresa della deambulazione in sole 24 ore. Poche settimane dopo, su quei "piedi blu", la ragazza è tornata a danzare, incentivata dai suoi medici all'importanza della piena ripresa della vita normale.

Questa vicenda ci dovrebbe indurre molta prudenza... Prudenza verso la tendenza spesso squisitamente americana di codificare diagnosi di condizioni post-infettive che potrebbero rischiare di cronicizzarsi solo perché gli viene attribuito un nome.

\section{Bibliografia}

1. Sacks O. Risvegli. Biblioteca Adelphi. Milano, 1987.

2. Rogers JP, Chesney E, Oliver D, et al. Psychiatric and neuropsychiatric presentations associated with severe coronavirus infections: a systematic review and meta-analysis with comparison to the Covid-19 pandemic. Lancet Psychiatry 2020;7(7):61 1-27. doi: 10.1016/S2215-0366(20)30203-0.

3. Mazza MG, De Lorenzo R, Conte $C$, et al. Anxiety and depression in Covid-19 survivors: role of inflammatory and clinical predictors. Brain Behav Immun 2020;89:594-600. doi: 10.1016/i.bbi.2020.07.037.

4. Steinberg L. II cervello adolescente: I'età delle opportunità. Feltrinelli, 2014.

5. Lerma LA, Chaudhary A, Bryan A, et al. Prevalence of autoantibody responses in acute coronavirus disease 2019 (Covid-19). J Transl Autoimmun 2020;3:100073. doi: 10.1016/i.jtauto. 2020.100073 .

6 . Huang $\mathrm{C}$, Huang $\mathrm{L}$, Wang $\mathrm{Y}$, et al. 6-month consequences of $\mathrm{Co}$ vid-19 in patients discharged from hospital: a cohort study. Lancet 2021;397(10270):220-32. doi: 10.1016/S0140-6736(20) $32656-8$.

7. Andina D, Noguera-Morel L, Bascuas-Arribas M, et al. Chilblains in children in the setting of Covid-19 pandemic. Pediatr Dermatol 2020;37(3):406-11. doi: 10.1111/pde.14215 [Epub ahead of print].

Francesca Peri', Alberto Tommasini ${ }^{1,2}$

'Dipartimento di Scienze Mediche, Chirurgiche e della Salute, Università di Trieste 1,2IRCCS Materno-Infantile "Burlo Garofolo", Trieste 